\title{
GASTROINTESTINAL DISTURBANCES IN RUNNERS
}

\author{
I. M. SHARMAN, PhD \\ Dunn Nutritional Laboratory, University of Cambridge
}

Richard Fogoros, a doctor at the Presbyterian University Hospital and the University of Pittsburgh School of Medicine, has recommended that physicians should recognise the various "abnormalities" that occur in healthy runners, either as physiological adaptations to physical training or as normal responses to extreme exertion. Such abnormalities include various bradyarihythmias, left ventricular enlargement, proteinuria and haematuria.

Conspicuously absent from medical literature is a description of the gastrointestinal symptoms experienced by many runners. Such complaints are usually relatively mild, consisting of abdominal cramping, bloating, and frequent watery bowel movements. These symptoms occur either during a period of rapid increase in daily running mileage, or during a particularly severe running episode, such as a highly competitive race. Sometimes these symptoms are sufficiently bothersome to the runner for him to seek advice from his doctor. Thus a 24-year-old medical student and long-distance runner is reported to have had a history of bloody diarrhoea. He complained of three weeks of abdominal cramping and watery diarrhoea that occured after hard runs, and for one week he noted bouts of dark-red blood mixed in with loose stools. His history was normal except for occasional episodes of abdominal cramping and watery diarrhoea for one year, since he first began running seriously. No cause was found for his condition, but his subsequent course of rare episodes of bloody diarrhoea only after severe exertion suggested running as the source.

How then does running cause gastrointestinal symptoms? One explanation is increased parasympathetic tone in runners. This is thought to be the cause of bradyarrhythmias seen in well trained individuals. Increased parasympathetic tone is maintained on a long-term basis, however, and would not explain why gastrointestinal symptoms decrease as training is maintained, or why symptoms increase with extreme exertion, when sympathetic tone is high.
Fogoros suggests a more likely explanation is relative gut ischaemia. During maximal exercise, whether an individual is well trained or not, blood flow to the gut is reduced by as much as $\mathbf{8 0}$ per cent, i.e. of the same range as seen in hypovolaemic shock. During prolonged severe exercise, as in a marathon, the athlete has the additional stress of hyperthermia, often in excess of $41^{\circ} \mathrm{C}$, which would predispose to ischaemic necrosis. It was in this setting, gut ischaemia and hyperthermia, that bloody diarrhoea developed in the above case.

Cardiovascular training causes changes in regional blood flow during submaximal exercise such that for a given level of submaximal work, blood flow towards the gut is much less reduced in the relatively untrained individual. In other words, for the same level of submaximal running, an athlete will have more gut ischaemia before he is well trained than he will afterwards. Such a training-induced change in regional blood flow could explain why runners have these gastrointestinal upsets when they start to increase their running and why the symptoms decrease after the training effect has taken hold. Relative gut ischaemia is thus a likely explanation for the gastrointestinal symptoms that athletes experience either after severe exertion or during an increase in training.

\section{HIGH ASCORBIC ACID INTAKE}

C. J. Hoyt of La Mesa, California has drawn attention to another source of milder runners' diarrhoea. Thus many runners have been advised that generous doses of vitamin C, $1 \mathrm{~g}$ or more daily, will lessen musculoskeletal symptoms. Such large doses of the vitamin frequently produce diarrhoea, the source of which may remain unrecognised for a long time. Physicians should therefore be alerted to this possible cause when such symptoms are presented to them. The advice to consume such large doses of vitamin C may well be contrasted with the view expressed earlier that "indiscriminate dosing with large quantities of ascorbic acid should not be allowed until the practice is shown to be harmless beyond all reasonable doubt."

\section{REFERENCES}

Fogoros, R. N., 1980 “Runners' trots: Gastrointestinal disturbances in runners”. J.Am.Med.Assoc. 243: $1743-4$. Hoyt, C. J., 1980 “Diarrhoea from vitamin C". J.Am.Med.Assoc. 244: 1674.

Anon, 1972 “Notes and comments: Vitamins in illness". Br.Med.J. 4: 786. 